\title{
The Challenges of Treating Lipoblastoma in the Sublingual Space - A Case Report
}

\author{
Sujesh Sreedharan ${ }^{1 *}$, Palasuntharam Shanmuhasuntharam ${ }^{2}$
}

\section{KEYWORDS}

Lipoblastoma, sublingual space, submandibular space

\begin{abstract}
The presence of lobulated tumour arising from immature adipose tissue is called lipoblastoma when occurring as localised or discrete lesion, and lipoblastomatosis when spread diffusely. The purpose of this case study is to report a case of an 18 - year old man who presented with lipoblastoma in the left sublingual space which was found to be in close proximity with the left sublingual gland. Magnetic resonance imaging (MRI) was taken and showed the tumour was confined within the sublingual space and no penetration was noted inferiorly through the mylohyoid muscle. Tumour dimension measured clinically was $3 \mathrm{~cm} \times 2 \mathrm{~cm} \times 2 \mathrm{~cm}$ and the anterior margin of the tumour crossed the midline and entered into the right sublingual space. Complete excision with sparing of neurovascular bundle was performed under general anaesthesia. Follow-up of 2 months showed complete remission of the lesion without any complications.
\end{abstract}

\section{INTRODUCTION}

Adipocytic tumours are a subtype of soft tissue tumors representing a complex group of lesions that may show a broad range of differentiation. They may be divided into benign, intermediate and malignant. Less than $10 \%$ of soft tissue neoplasms in the 1st 2 decades of life have an adipose phenotype and most are benign [1]. The most common are various types of lipoma and lipoblastoma. Liposarcoma in young patients is rare and has a distinctive distribution of histologic subtypes, including classic myxoid liposarcoma, and unusual variants, such as pleomorphic-myxoid liposarcoma [2]. Lipoblastoma is classified under benign neoplasm arising from the embryonal white fat. Lipoblastoma was first described by Jaffe in 1926 [3]. It was not identified as a distinctive tumour until Vellios et al. discovered that the adipose tissue of three-week embryos which

${ }^{1}$ Oral and Maxillofacial Surgery Department, Hospital Enche Besar Hajjah Khalsom, Kluang, Johor, Malaysia

${ }^{2}$ Department of Oral and Maxillofacial Clinical Sciences, Faculty of Dentistry, University of Malaya, Kuala Lumpur, Malaysia

*Correspondence: sujeshsree@yahoo.co.uk disappeared later in foetal development that was the basis for the pathogenesis of lipoblastoma [4]. It is an uncommon tumour which affects almost exclusively infants and young children below the age of 12 years [5]. Anatomic sites usually involved include extremities, neck, trunk, retroperitoneum and mediastinum [6]. Occurrence of lipoblastoma in the oral cavity is rare which corresponds to less than $1.7 \%$ of all benign oral soft tissue tumours [7]. They commonly present as slow growing asymptomatic lesions with a characteristic yellow colour, soft doughy feel and lobulated [5].

To our knowledge, there are very few studies done on lipoblastoma found in the oral cavity. There is no gender predilection. Some studies, however, have shown a male predominance [2]. Although benign in nature, their progressive growth may cause interference with speech and mastication due to tumour's dimension.

\section{CASE REPORT}

An 18-year-old man presented to the Oral and Maxillofacial Surgery Department, University of 
Malaya with a painless swelling at the floor of the mouth on the left side of 2 months duration. Initially, the swelling was pea-sized which gradually grew to the present size. There was no history of pain or increase in size of the swelling during mastication. However, patient did complain of having discomfort while chewing food. Besides this, his speech was also disturbed due to abnormal positioning of his tongue caused by the swelling.

Further history revealed that he previously experienced the same problem but on the contralateral side. He came 7 years earlier with a chief complaint of right - sided facial asymmetry with obvious swelling over the right submandibular region. An incisional biopsy intraorally was done and sent for histopathology examination (HPE) which later interpreted as lipoblastoma. MRI taken at that time revealed hyper-intensity suggesting the presence of fat lobules at the right sublingual space and breaching through the mylohyoid muscle to enter into the submandibular space. Patient underwent total excision of the lipoblastoma along with the removal of right sublingual and submandibular glands under general anesthesia in 2010. Healing was uneventful and patient had no complaints thereafter.

At present, his medical, family and personal histories were not contributory. On general assessment, patient was moderately built and wellnourished. Extraorally, his face was symmetrical with no obvious swelling. Intraoral examination revealed a single dome shaped swelling. The swelling had smooth texture, compressible but not reducible, non-pulsating and non-tender to palpation. The margins of the swelling were slippery under the palpating finger. Left Wharton's duct was patent with evidence of normal saliva flow. Considering all the clinical features, the differential diagnoses considered were of lipoma, lipoblastoma, ranula, dermoid cyst, salivary gland tumour and liposarcoma. Aspiration was carried out, but no fluid could be detected. Other than that, there was no odontogenic source of infection and this was confirmed radiographically. Routine blood investigation was found to be normal. We managed to obtain MRI of the neck to observe for the extent of the lesion.

MRI showed a well circumscribed lesion arising from the left sublingual region anteriorly at floor of the mouth measuring approximately $1.5 \mathrm{~cm} \times 3.6$ $\mathrm{cm} \times 2.6 \mathrm{~cm}$ (Figure 1). The lesion crosses the midline with the larger component noted on the left displacing the ventrum of the tongue superiorly. There was no evidence of adjacent bone erosion or extension of the lesion into extrinsic tongue muscles. Our clinical diagnosis at this stage was recurrent lipoblastoma in the left sublingual space since patient had a similar clinical presentation 7 years ago on the contralateral side which was confirmed with HPE post-surgery. Surgical excision of the current lesion was done under general anesthesia (Figure 2).

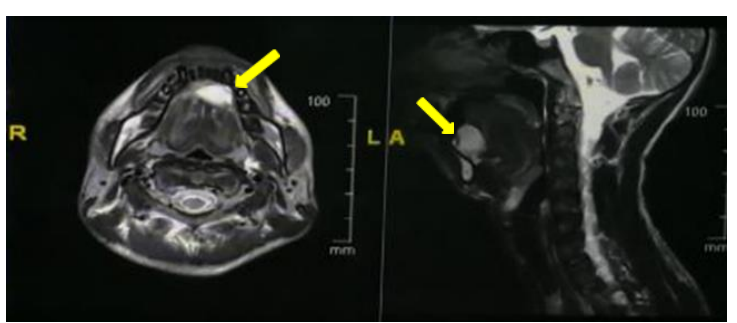

Figure $1 \mathrm{MRI}$ showing lesion (arrow) in the sublingual space.
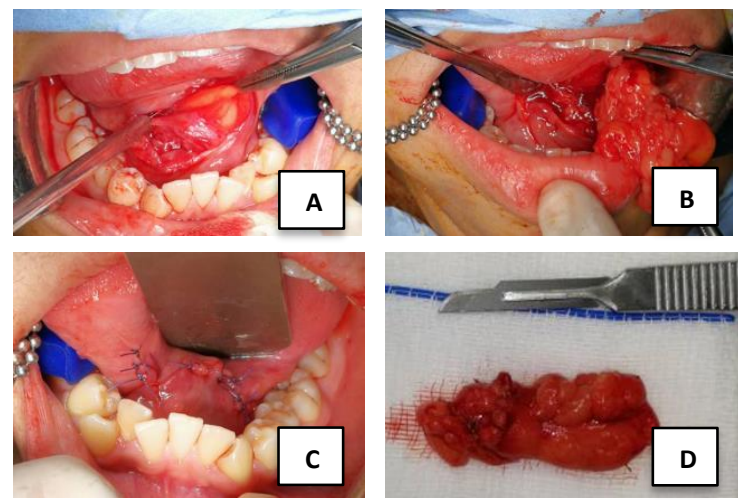

Figure 2 A) Submucosal incision was made above the left Wharton's duct. Care was taken not to injure the duct (arrow); B) with blunt dissection; the lesion was separated from the surrounding mucosa. The lesion was excised in toto without damaging any neurovascular bundle; C) Mucosa was repositioned and sutured with Vicryl 4/0; D) Surgical specimen was sent for HPE.
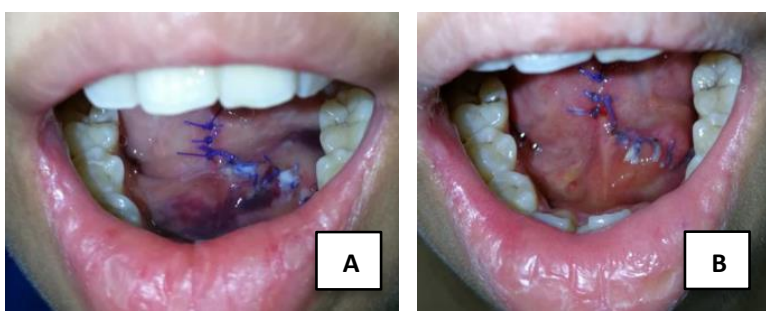

Figure 3 A) Post-operative day 1, showed hematoma of the surgical site but there was no sign of impending airway obstruction due to the swelling; B) Post - operative day 7 showing clinically good recovery of the surgical site. Left Wharton's orifice showed positive sign of saliva flow.

Our histopathological findings were similar to those reported in various published studies. Histologically, characteristic lobular architecture is 
seen with sheets of adipocytes separated by fibrovascular septa. The adipocytes demonstrate different stages of maturation from primitive spindle cells to vacuolated lipoblasts to mature adipocytes in varying proportions. The surgical specimen was consistent with our clinical diagnosis of lipoblastoma.
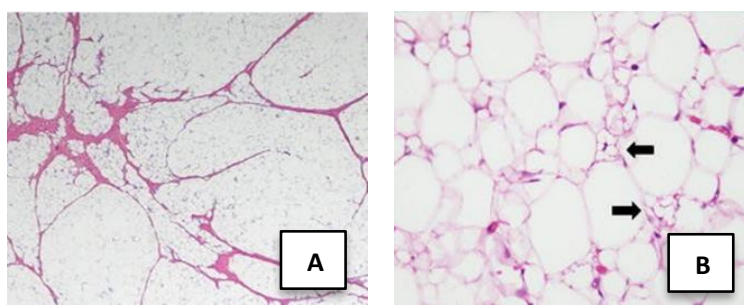

Figure 4 A) Microscopic features of lipoblastoma. Fibrous septations divide the tumour into lobules; B) Variable sized multivacuolated lipoblasts (arrows) admixed with adipocytes

\section{DISCUSSION}

Lesion found in the floor of the mouth can be very challenging to diagnose and to manage. There could be many possible lesions arise within the corresponding structures found within the sublingual or submandibular spaces that form the floor of the mouth. A common diagnosis of ranula for a swelling can be misleading if a thorough investigation and appropriate imaging is not included as part of the treatment management. Table 1 tabulates all the possible lesions that may arise from the floor of the mouth [8].

Table 1 Possible lesions in the floor of the mouth

\begin{tabular}{ll}
\hline $\begin{array}{l}\text { Inflammatory / } \\
\text { trauma }\end{array}$ & $\begin{array}{l}\text { Mucus retention phenomenon } \\
\text { (Ranula) } \\
\text { Mucus extravasation phenomenon } \\
\text { (Sialocele) }\end{array}$ \\
\hline Developmental & $\begin{array}{l}\text { Epidermoid cyst } \\
\text { Dermoid cyst } \\
\text { Lymphoepithelial cyst }\end{array}$ \\
Neoplasm & $\begin{array}{l}\text { Lipoma } \\
\text { Liposarcoma }\end{array}$ \\
& Lipoblastoma/ lipoblastomatosis \\
& Salivary gland tumour \\
\hline
\end{tabular}

As shown by our case report, although lipoblastoma is most often found superficially at the subcutaneous or submucosal level, careful consideration need to be given if it is found at the sublingual space in the oral cavity. This space is bounded by the hyoglossus muscle of the tongue medially, laterally by the mandible, posteriorly by the muscles along the base of the tongue, superiorly by the mucosa of the floor of the oral cavity and inferiorly by the mylohyoid muscles.
Sublingual space contains the hypoglossal nerve, lingual nerve, sublingual glands, and deep part of the submandibular gland which is continuous along the posterior free border of the mylohyoid muscle, submandibular duct and lingual vein. An important anatomic consideration in surgery of the floor of the mouth is the submandibular duct which runs anteriorly to open into the sublingual caruncle, passing from laterally to medially as there is lingual nerve which passes it and loops under the duct.

A proper informed consent must be taken from patients who will be undergoing surgery in the floor of the mouth. Surgical complications such as nerve disturbances causing temporary or permanent sensory disability of the tongue, formation of ranula, salivary ductal injury causing reduced moisture in the mouth, severe haemorrhages and swelling requiring airway protections need to emphasized prior to surgery.

Oral adipose tissue neoplasm like lipoblastoma may sometimes infiltrate the muscles and become fixed to the surrounding structures within the sublingual space [5]. Nerve injuries are an inherent risk of any surgery despite the best of care provided. The patient with a nerve injury may experience a variety of sensations, such as numbness, tingling, burning, crawling sensations, electric shocks or hypersensitivity of the affected area. These sensations may interfere with normal chewing, drinking of liquids, speaking, shaving or kissing and they are distressing to the patient. Such symptoms, if persistent beyond several months following the initial injury, may indicate a nerve injury that will not resolve on its own and should be evaluated further [8]. Another possible surgical complication is mucous retention cyst or ranula which can result if part of the sublingual gland which was damaged causes obstruction to the secretory ducts. Patient may present with a bluish, cystic mass at floor of the mouth which may lead to lingual elevation or difficulty with deglutition [8].

In addition to these, clinician may need to be cautious about the vascular supply in the floor of the mouth. There is abundant rich anastomosing vascular supply that supplies the bed of the mouth arising from three main vessels, namely (i) the sublingual arteries, (ii) the submental arteries, and (iii) the incisive arteries. Although severe haemorrhage from this anastomosing plexus has not been reported as a complication of lesion removal from the floor of the mouth, care should be taken since the risk of potential life threatening complications, such as severe arterial or venous bleeding and large hematomas may occur [8]. 
The diagnostic work up for solitary lipoblastoma requires a proper history, examination and investigation including an appropriate radio imaging followed by treatment which includes exploration and wide excision of the lesion. The same line of management and treatment was carried out in our case. Lipoblastoma has an excellent prognosis. Complete but conservative local excision is effective treatment [9]. Majority of authors reviewed support preserving function in lieu of complete surgical resection. Factors contributing to this position include lack of malignant transformation, possible spontaneous regression, the ability to monitor tumor growth with $\mathrm{MRI}$, and 2 of the 5 reported recurrences occurred over 10 years after primary surgery [9]. Recurrence in up to half of cases is usually attributed to incomplete or subtotal excision, and recurrences can be successfully re-excised [1, 2]. Similarly in our case, recurrence occurs within 10 years from the primary surgery done at contralateral side, confirming the fact that a high recurrence rate of $14-25 \%$ despite surgical excision [10]. Non-malignant nature of lipoblastoma may cause compressive symptoms such as pain during mastication and swallowing due to the tentaclelike extensions enveloping critical anatomical structures [9]. This was not the case in the patient presented in this report, the tumor was asymptomatic, and there were no compressive symptoms.

\section{CONCLUSION}

Lipoblastoma is a rare benign paediatric neoplasm of embryonal white fat with little risk of recurrence following total surgical excision, but it may progress into local invasion or infiltration if not treated surgically. Patient may need to be cautioned regarding complications that may arise prior to surgery in the floor of the mouth. No adjuvant therapies are usually necessary once tumour is excised. Lesion is best diagnosed on histopathological studies following excision.

\section{STATEMENT OF INFORMED CONSENT}

Written informed consent was obtained from the patient for the publication of this case report and accompanying images. This case was approved for publication by the Faculty of Dentistry, University of Malaya, Kuala Lumpur, Malaysia.

\section{ACKNOWLEDGEMENT}

I would like to thank Associate Professor Shanmuhasuntharam for his help during preparation of this case report and constantly motivating me to work harder.

\section{DECLARATION OF INTEREST}

The authors report no conflicts of interest in this work.

\section{REFERENCES}

1. Akhtar T, Alladi A, Ahmed SM, Siddappa OS. Giant lipoblastoma of the thigh: a rare soft tissue tumor in an infant. J Cancer Res Ther. 2012; 8(1):157-8.

2. Coffin CM, Alaggio R. Adipose and myxoid tumors of childhood and adolescence. Pediatr Dev Pathol. 2012; 15(1 Suppl):239-54.

3. Kumar A, Brierley D, Hunter KD, Lee N. Rapidly-growing buccal mass in a 6-month-old infant. Br J Oral Maxillofac Surg. 2015; 53(9):888-90.

4. Vellios F, Baez J, Shumacker HB. Lipoblastomatosis: a tumor of fetal fat different from hibernoma. Report of a case with observations on the embryogenesis of human adipose tissue. Am J Pathol. 1958; 34(6):114959.

5. Fletcher CB JA, Hogendoorn PCW, Mertens F. WHO Classification of Tumours of Soft Tissue and Bone. Lyon, France: International Agency for Research on Cancer; 2013 World Health Organization Classification of Tumours. 2013; Vol 5. (4th ed.)

6. Creytens D. A contemporary review of myxoid adipocytic tumors. Semin Diagn Pathol. 2019; 36(2):129-41.

7. Shetty L, Kshirsagar K, Kulkarni D. Lipoma in the retromolar area: A case report with review. Med J DY Patil Univ 2012; 5:79-82

8. Shafer, W.G., Hine, M.K. and Levy, B.M. Text Book of Oral Pathology. 4th ed. West Washington Square, WB Saunders Company, Philadelphia. 1983. 
9. Pham NS, Poirier B, Fuller SC, Dublin AB, Tollefson TT. Pediatric lipoblastoma in the head and neck: a systematic review of 48 reported cases. Int J Pediatr Otorhinolaryngol. 2010; 74(7):723-8.

10. Chun YS, Kim WK, Park KW, Lee SC, Jung SE. Lipoblastoma. J Pediatr Surg. 2001; 36(6):905-7.

\section{Editorial History}

Date of Submission: 12 Dec 2020

Review \& Revision: 23 Dec 2020 - 28 May 2021

Accepted: 9 June 2021

Published: 23 July 2021

License Information: This work is licensed under a Creative Commons Attribution 